\title{
EM Specimen Preparation of Nanoparticles Using Ultrasonic Spray Technique
}

\author{
L.-C. Lai ${ }^{1}$, W.-A. Chiou ${ }^{1}$, S.-C. Tsai ${ }^{2}$, Yu L. Song ${ }^{3,4}$, Y.-F. Chou ${ }^{3}$, C.-S. Tsai ${ }^{4}$
}

1. Dept. of Chem. Engineering and Materials Science, University of California, Irvine, CA 92697

2. Dept. of Chemical Engineering, California State University, Long Beach, CA 90840

3. Dept. of Mechanical Engineering, National Taiwan University, Taipei, Taiwan, Republic of China

4. Dept. of Electrical Engineering and Computer Science, Univ. of California, Irvine

Particles dispersing technology is critical in nanoparticles research. The successful application of nanoparticles in a particular field often depends upon the ability to properly disperse the nanoparticles. Well-dispersed particles enable some traditional difficulties on size analysis to become possible, such as size distribution of nanoparticle as well as for the observation of individual clay particles in an electron microscope [1]. Several researchers have been reported various methods to analyze the distribution of disperse nanoparticle size by electron microscopy. However, the aggregation of small particles upon drying remains a serious problem during the specimen preparation for electron microscopy. In the present research work, the unique technique has been developed to disperse the nanoparticles uniformly using ultrasonic atomization spray technique.

The atomizer is a MEMS-based $0.5 \mathrm{MHz}$ ultrasonic nozzle that made up of a piezoelectric drive section and a 3-Fourier horn silicon-resonator with a central channel for liquid flow [2]. Nano-size $\mathrm{Au}$ particles purchased from a commercial vendor and selected purified clay minerals (smectite) were used for the experiment. As the Au or clay particle suspension is pumped into the special channel, a thin film of liquid forms at the nozzle tip that vibrates at the resonant frequency of 484.5 $\mathrm{kHz}$. The ultrasonic vibration break up the volume of liquid and spray the suspension onto a substrate. Si and carbon films were used to investigate the possible surface effect of substrate on different nanoparticles.

SEM image, as shown in Fig. 1a, shows that atomized/sprayed gold particles on Si substrate are very well dispersed and uniformly distributed. Almost no agglomeration of Au particles can be found. Although often 50-60 Au particles may group together, no Au aggregates were observed. However, if the same $\mathrm{Au}$ suspension sprayed on a $\mathrm{C}$ thin film the particle distribution pattern was quite different. TEM image reveals that 20-30 Au particles tend to from a "chain-like" pattern on the C substrate though few agglomerates were seen (Fig. 1b). The different distribution pattern of $\mathrm{Au}$ particles on two types of substrates may be resulted from the different surface properties of substrates. Uniform dispersion of clay particles on substrate has been a very difficult task in clay research. Large agglomerates with overlapped clay particles are typical in TEM images that obtained from conventional specimen preparation technique (Fig. 2a), and the characterization of clay particle shape and size is nearly impossible. However, with the new atomization spray technique, clays are much better dispersed and different orientation can also be observed. Both TEM (Fig. 2b) and SEM (Fig. 3) images illustrate the good dispersion of clay particles on $\mathrm{C}$ thin film though some very thin clay aggregates are still visible in the present investigation. The improved results indicate that further research with other spray parameters (such as concentration of clay suspension and spray time) shall be able to solve this problem.

[1] W. A. Chiou, et. al., ICEM -15, Durban, S.A., p.937-938, 2002.

[2] S.C. Tsai et. al., IEEE Trans. on UFFC, 51, p.277-286, 2004. 


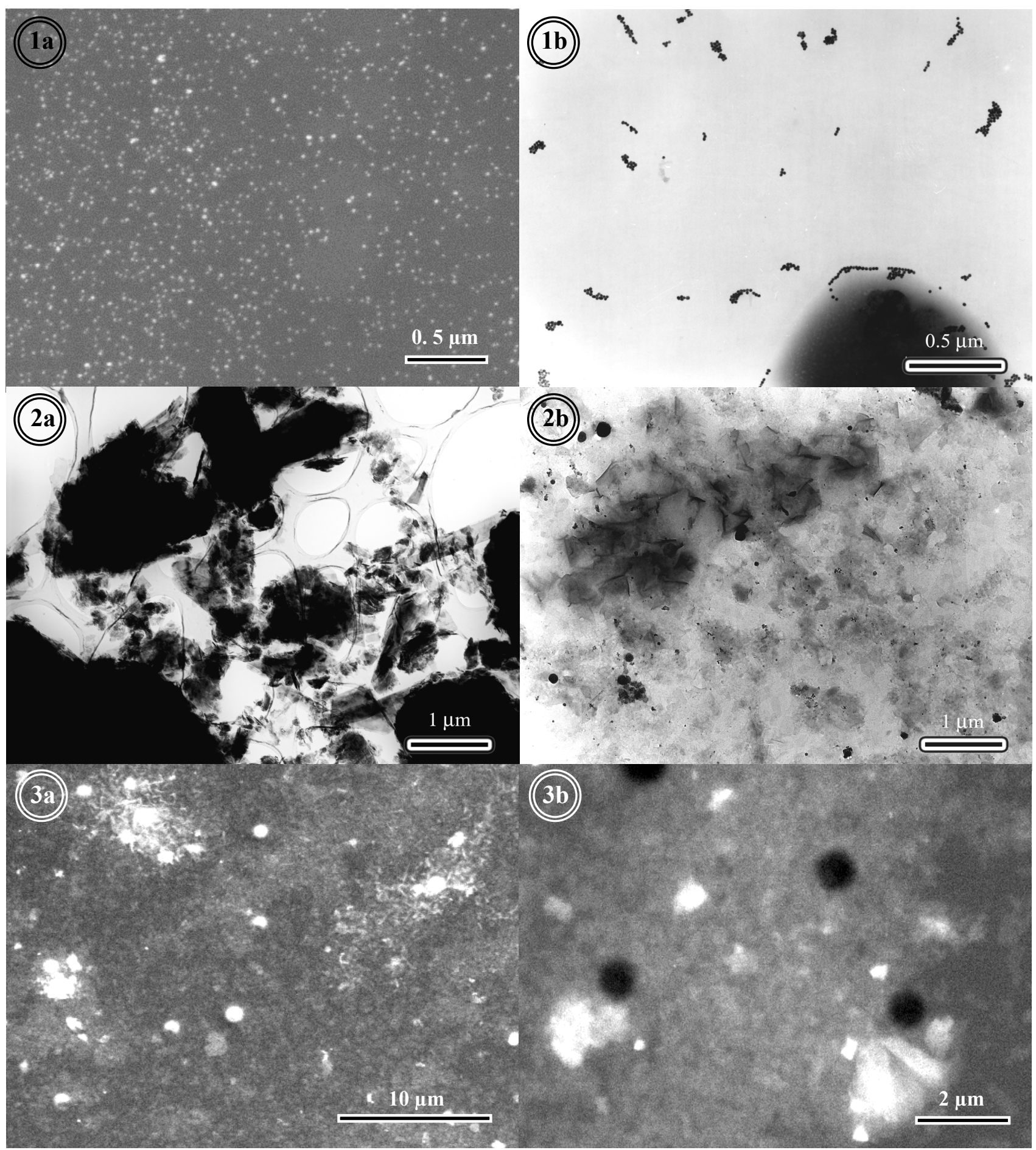

Fig. 1. (a) SEM image of Au particles on Si substrate showing the good dispersion.

(b) TEM image of Au particles on C thin film revealing "chain-like" feature.

Fig. 2. (a) TEM image depicting typical clay aggregates on $\mathrm{C}$ thin film by conventional method.

(b) TEM image of atomized spray of clay on $\mathrm{C}$ film revealing better dispersed clay particles.

Fig. 3. (a) Low magnification SEM image depicting dispersed clay particles on Si substrate.

(b) High magnification SEM image showing dispersed and uniformly distributed clays. 